\title{
On the Value of Look-Ahead in Competitive Online Convex Optimization
}

\author{
Ming Shi \\ School of Electrical and Computer \\ Engineering \\ Purdue University \\ West Lafayette, IN, USA \\ sming@purdue.edu
}

\author{
Xiaojun Lin \\ School of Electrical and Computer \\ Engineering \\ Purdue University \\ West Lafayette, IN, USA \\ linx@ecn.purdue.edu
}

\author{
Lei Jiao \\ Department of Computer and \\ Information Science \\ University of Oregon \\ Eugene, OR, USA \\ jiao@cs.uoregon.edu
}

\begin{abstract}
Although using look-ahead information is known to improve the competitive ratios of online convex optimization (OCO) problems with switching costs, the competitive ratios obtained from existing results often depend on the cost coefficients of the problem, and can potentially be large. In this paper, we propose new online algorithms that can utilize look-ahead to achieve much lower competitive ratios for OCO problems with switching costs and hard constraints. For the perfect look-ahead case where the algorithm is provided with the exact inputs in a future look-ahead window of size $K$, we design an Averaging Regularized Moving Horizon Control (ARMHC) algorithm that can achieve a competitive ratio of $\frac{K+1}{K}$. To the best of our knowledge, ARMHC is the first to attain a low competitive ratio that is independent of either the coefficients of the switching costs and service costs, or the upper and lower bounds of the inputs. Then, for the case when the future look-ahead has errors, we develop a Weighting Regularized Moving Horizon Control (WRMHC) algorithm that carefully weights the decisions inside the look-ahead window based on the accuracy of the look-ahead information. As a result, WRMHC also achieves a low competitive ratio that is independent of the cost coefficients, even with uncertain hard constraints. Finally, our analysis extends online primal-dual analysis to the case with look-ahead by introducing a novel "re-stitching" idea, which is of independent interest.
\end{abstract}

\section{CCS CONCEPTS}

- Theory of computation $\rightarrow$ Online algorithms; • Mathematics of computing $\rightarrow$ Convex optimization; $・$ Networks $\rightarrow$ Network algorithms.

\section{KEYWORDS}

Online convex optimization (OCO); Look-ahead; Competitive analysis; Online primal-dual analysis

\section{ACM Reference Format:}

Ming Shi, Xiaojun Lin, and Lei Jiao. 2019. On the Value of Look-Ahead in Competitive Online Convex Optimization. In ACM SIGMETRICS / International Conference on Measurement and Modeling of Computer Systems

Permission to make digital or hard copies of part or all of this work for personal or classroom use is granted without fee provided that copies are not made or distributed for profit or commercial advantage and that copies bear this notice and the full citation on the first page. Copyrights for third-party components of this work must be honored. For all other uses, contact the owner/author(s).

SIGMETRICS '19 Abstracts, fune 24-28, 2019, Phoenix, AZ, USA

(C) 2019 Copyright held by the owner/author(s).

ACM ISBN 978-1-4503-6678-6/19/06.

https://doi.org/10.1145/3309697.3331474
(SIGMETRICS '19 Abstracts), fune 24-28, 2019, Phoenix, AZ, USA. ACM, New York, NY, USA, 2 pages. https://doi.org/10.1145/3309697.3331474

\section{INTRODUCTION}

In this paper, we study online convex optimization (OCO) problems with switching costs and hard constraints [1-4]. In its generic form, an OCO problem proceeds in time $t=1,2, \ldots, \mathcal{T}$. At each time $t$, first the environment (or adversary) reveals the input $\vec{A}(t) \in$ $\mathbb{R}^{M \times 1}$ and the service-cost function $h_{t}(\cdot, \vec{A}(t))$. Then, the decision maker chooses a decision $\vec{X}(t) \in \mathbb{R}^{N \times 1}$ from a convex decision set $\mathbb{X}_{t}(\vec{A}(t)) . \mathbb{X}_{t}(\vec{A}(t))$ may contain hard constraints that depend on input, and can be written as linear inequalities in $(\vec{X}(t), \vec{A}(t))$, i.e., $\boldsymbol{B}_{1} \vec{X}(t) \geq \boldsymbol{B}_{2} \vec{A}(t)$, where $\boldsymbol{B}_{1} \geq 0$ is an $L \times N$ matrix and $\boldsymbol{B}_{2} \geq 0$ is an $L \times M$ matrix. Provided that the hard constraint is met at time $t$, a linear service cost $h_{t}(\vec{X}(t), \vec{A}(t))=\vec{C}^{\mathrm{T}}(t) \vec{X}(t)$ is incurred for the decision $\vec{X}(t)$, where $\vec{C}(t) \in \mathbb{R}^{N \times 1}$ is the service-cost coefficient. (Some of our results will also be generalized to convex service costs.) Note that if the hard constraint is not satisfied, we assume that the service cost will be $+\infty$. Additionally, there is a switching cost $\vec{W}^{\mathrm{T}}[\vec{X}(t)-\vec{X}(t-1)]^{+}$that penalizes the increment of each entry of the decision $\vec{X}(t)$ at time $t$, where $\vec{W} \in \mathbb{R}^{N \times 1}$ is the switching-cost coefficient and $[x]^{+}=\max \{x, 0\}$. Such an OCO formulation can be used to model online problems in many application domains, such as machine learning, networking, cloud computing, cyber-physical systems and wireless systems [1-4].

In this paper, we focus on the setting where the decision maker must make decisions in an online fashion. This means that at each time $t$, she must make the current decision $\vec{X}(t)$ based on only the current input $\vec{A}(t)$ and a limited amount of future look-ahead. Moreover, once the decision $\vec{X}(t)$ is made, the decision is irrevocable. Thus, this problem becomes an online problem. In contrast, in an offline setting, the entire input sequence $\vec{A}(1)$ to $\vec{A}(\mathcal{T})$ was known in advance. Then, the optimal offline decisions could be found by solving a simple convex optimization problem. Our goal is then to design online algorithms with low competitive ratios, where the competitive ratio is the worst-case ratio between the total cost of an online algorithm and that of the optimal offline solution.

Although there exist a number of online algorithms that can achieve provable competitive ratios for fairly general classes of OCO problems $[1,3,4]$, it remains a challenge to achieve low competitive ratios. For example, the regularization method [1] can be used on OCO problems without any future information. While a provable competitive ratio can be shown, the resulting competitive ratio often depends on the dimension of the problem and the 
characteristics of the inputs, and can potentially be large. This is not surprising because, when there is absolutely no future knowledge, the competitive ratio often has to be large due to adversarial inputs. Many subsequent studies thus focus on the setting with a limited amount of future prediction, in the form of a look-ahead window [2,3]. Intuitively, the further ahead such prediction is provided, and the more accurate the prediction in the look-ahead window is, the lower the competitive ratio should be. For the case with perfect look-ahead, Lin et al. [3] propose Averaging Fixed Horizon Control (AFHC), which is shown to be $\left(1+\max _{n} \frac{w_{n}}{(K+1) \cdot c_{n}}\right)$ competitive, where $K$ is the size of the look-ahead window, $c_{n}$ is the service-cost coefficient and $w_{n}$ is the switching-cost coefficient. Note that this competitive ratio of AFHC is indeed decreasing in $K$. However, it still increases with $\max _{n} \frac{w_{n}}{c_{n}}$, and thus can potentially be very large. Therefore, for the OCO problems that we consider in this paper, it remains an open question whether one can develop online algorithms that can utilize perfect look-ahead to achieve low competitive ratios both decreasing in $K$ and independent of the service-cost and switching-cost coefficients. For the case with imperfect look-ahead, existing results mainly focus on the competitive difference [2]. Unfortunately, even if the competitive difference is bounded, it does not imply that the competitive ratio is bounded. In fact, even the competitive difference in [2] still depends on such problem parameters as the coefficients of the switching costs. Further, these existing results typically do not consider hard constraints that depend on the uncertain inputs. Thus, for the general OCO problems that we study in this paper, it also remains an open problem to develop online algorithms with low competitive ratios under imperfect look-ahead and uncertain hard constraints.

\section{MAIN RESULTS}

\subsection{Perfect Look-Ahead}

We first study the case with perfect look-ahead, where at time $t$, the precise value of inputs from $\vec{A}(t)$ to $\vec{A}(t+K)$ are given to the decision maker. We first show, via a simple counter-example, that AFHC could still incur large competitive ratios. This counter-example thus motivates us to develop new competitive online algorithms that can achieve significantly lower competitive ratios with perfect look-ahead.

Then, we develop a novel Averaging Regularized Moving Horizon Control (ARMHC) algorithm that is $\left(\frac{K+1}{K}\right)$-competitive. We note that this result is highly appealing because the competitive ratio depends only on the size of the look-ahead window $K$, but not on other problem parameters, including both the coefficients of the service costs and switching costs, and the upper and lower bounds of future inputs. To the best of our knowledge, this is the first result of such type in the literature for general OCO problems with perfect look-ahead. Further, note that even with a small lookahead window of size $K=1$, the competitive ratio is already 2 . This suggests that even a small window of perfect look-ahead can be highly effective in reducing the competitive ratio. Moreover, as the look-ahead window size $K$ increases, the competitive ratio of ARMHC further decreases to 1 .

In order to develop this result, we introduce in ARMHC two key ideas. One is to use a "regularization term" at the tail of each look-ahead window, and the other is to commit to only the first $K-1$ decisions in the look-ahead window. As we will elaborate in Sec. 3.2 of our full paper [5], both ideas are crucial for achieving competitive ratios much lower than the state-of-art. In the proof, we introduce a novel "re-stitching" idea to extend online primal-dual analysis [1] to the case with look-ahead, which is of independent interest.

\subsection{Imperfect Look-Ahead}

We next study the case with imperfect look-ahead, where at time $t$, only a possible trajectory of the near-future inputs from $\vec{A}(t+1)$ to $\vec{A}(t+K)$ is given to the decision maker, as well as upper and lower bounds of the possible future inputs.

We first propose the Averaging Regularized Moving Horizon Control-Imperfect Look-ahead (ARMHC-IL) algorithm, which uses the upper bound of the future inputs so that future input-dependent hard constraints can always be satisfied. We show that the competitive ratio of ARMHC-IL is $\frac{K+1}{K} \cdot \max _{\{i \in[0, K-1], m \in[1, M]\}} \frac{f_{m}^{\text {up }}(i)}{f_{m}^{\text {low }}(i)}$, where $f_{m}^{\text {up }}(i)$ and $f_{m}^{\text {low }}(i)$ are upper bounds and lower bounds, respectively, of the relative prediction error for the $i$-th time slot in the look-ahead window. We note that this competitive ratio of ARMHCIL is highly sensitive to the worst-case prediction error. This is mainly because ARMHC-IL treats all time slots uniformly. Knowing the prediction accuracy of each future time slot, we could have treated the different time slots differently, according to how reliable the prediction is. We then propose Weighting Regularized Moving Horizon Control (WRMHC), which assigns different weights to the costs and decisions at different time slots. By choosing the optimal weights $\rho^{*}(i)=\min _{m \in[1, M]} \frac{f_{m}^{\text {low }}(i)}{f_{m}^{\text {up }}(i)}$ and the optimal committed window size $k^{*}$, we show that the competitive ratio of WRMHC is $\frac{k^{*}+1}{k^{*}-1}$. Note that this competitive ratio of WRMHC is $1+\sum_{i=1} \min _{m \in[1, M]} \frac{f_{m}(i)}{f_{m}^{\mathrm{up}}(i)}$

independent of the service-cost and switching-cost coefficients and is less sensitive to the worst-case prediction error than ARMHC-IL.

The full paper is available at [5].

\section{ACKNOWLEDGMENTS}

This work has been supported in part by NSF grants ECCS-1509536 and CNS-1717493.

\section{REFERENCES}

[1] Niv Buchbinder, Shahar Chen, and Joseph (Seffi) Naor. 2014. Competitive Analysis via Regularization. In Proceedings of the 25th Annual ACM-SIAM Symposium on Discrete Algorithms (SODA '14). Society for Industrial and Applied Mathematics, Philadelphia, PA, USA, 436-444.

[2] Niangjun Chen, Joshua Comden, Zhenhua Liu, Anshul Gandhi, and Adam Wierman. 2016. Using Predictions in Online Optimization: Looking Forward with an Eye on the Past. ACM SIGMETRICS Performance Evaluation Review 44, 1 (2016), 193-206.

[3] Minghong Lin, Zhenhua Liu, Adam Wierman, and Lachlan LH Andrew. 2012. Online Algorithms for Geographical Load Balancing. In International Green Computing Conference (IGCC). IEEE, 1-10.

[4] Ming Shi, Xiaojun Lin, Sonia Fahmy, and Dong-Hoon Shin. 2018. Competitive Online Convex Optimization with Switching Costs and Ramp Constraints. In IEEE Conference on Computer Communications (INFOCOM). 1835-1843.

[5] Ming Shi, Xiaojun Lin, and Lei Jiao. 2019. On the Value of Look-Ahead in Competitive Online Convex Optimization. Proceedings of the ACM on Measurement and Analysis of Computing Systems (POMACS) 3, 2 (2019). 